\title{
Resource Allocation for Cognitive Satellite Uplink and Fixed-Service Terrestrial Coexistence in Ka-band
}

\author{
Eva Lagunas ${ }^{1}$, Shree Krishna Sharma ${ }^{1}$, Sina Maleki ${ }^{1}$, Symeon Chatzinotas ${ }^{1}$, \\ Joel Grotz ${ }^{2}$, Jens Krause ${ }^{3}$, and Björn Ottersten ${ }^{1}$ \\ 1 Interdisciplinary Centre for Security, Reliability and Trust (SnT), University of \\ Luxembourg \\ \{eva.lagunas, shree.sharma, sina.maleki, symeon. chatzinotas, bjorn. \\ ottersten\}@uni.lu, \\ 2 Technical Labs, Newtec, Belgium, joel.grotz@newtec.eu, \\ ${ }^{3}$ SES, Betzdorf, Luxembourg, jens.krause@ses.com
}

\begin{abstract}
This paper addresses the cognitive Geostationary Orbit (GSO) satellite uplink where satellite terminals reuse frequency bands of FixedService (FS) terrestrial microwave links which are the incumbent users in the $\mathrm{Ka}$ 27.5-29.5 GHz band. In the scenario considered herein, the transmitted power of the cognitive satellite user has to ensure that the interference impact on potentially present FS links does not exceed the regulatory interference limitations. In order to satisfy the interference constraint and assuming the existence of a complete and reliable FS database, this paper proposes a Joint Power and Carrier Allocation (JPCA) strategy to enable the cognitive uplink access to GSO Fixed Satellite Service (FSS) terminals. The proposed approach identifies the worst FS link per user in terms of interference and divides the amount of tolerable interference among the maximum number of FSS terminal users that can potentially interfere with it. In so doing, the cognitive system is guaranteed to never exceed the prescribed interference threshold. Subsequently, powers and carriers are jointly allocated so as to maximize the throughput of the FSS system. Supporting results based on numerical simulations are provided. It is shown that the proposed cognitive approach represents a promising solution to significantly boost the performance of conventional satellite systems.
\end{abstract}

\section{Introduction}

The Digital Agenda for Europe foresees broadband interactive access as a cornerstone in the recovery and development plan for Europe [1]. The challenging objectives set forth by the European Commission are to provide basic broadband access to all Europeans by 2013 (at least $30 \mathrm{Mbps}$ ) and to ensure that at least $50 \%$ of the households get $100 \mathrm{Mbps}$ by 2020 [2]. In this context, Satellite Communications (SatCom) can play a key role to ensure ubiquitous coverage including remote and rural regions, where terrestrial deployment cannot be guaranteed or its cost is prohibitive. Traditional single-beam satellites operating in 


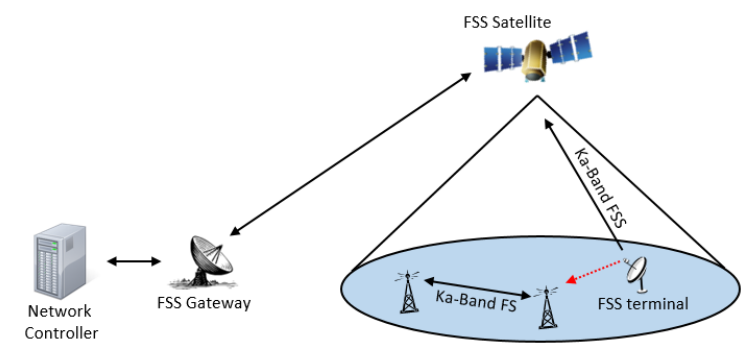

Fig. 1: Spectral coexistence of FSS uplink with the FS terrestrial link in the Ka-band (27.5-29.5 GHz)

$\mathrm{C} / \mathrm{Ku}$-band show limitations in supporting the flexible distribution of bandwidth needed for broadband applications [3,4]. Novel multi-beam satellites operating in the Ka-band frequency spectrum have recently gained attention among satellite telecommunication industries because they can significantly enhance the system capacity as a result of higher frequency reuse and multiple narrowly focused beams. Examples of high throughput satellites operating in Ka-band are Eutelsats KaSat [5], VIASAT 1 [6] and SES-12 [7].

The provision of widely available and competitively-priced broadband satellite services critically depends on the availability of radio spectrum resources. Spectrum congestion has been identified as the main limiting factor in providing solutions meeting the user expectations by 2020 [8]. Cognitive Radio (CR) [9] is conceived as an ideal spectrum management tool to solve the spectrum scarcity, as it enables unlicensed systems to opportunistically utilize the underutilized licensed bands. As far as cognitive SatCom is concerned, recently a number of research projects have been undertaken to address the spectrum sharing concept between two satellite systems or between satellite and terrestrial systems [10-13]. Within [10], three scenarios have been identified as potentially positively impacted by the use of CR techniques. In this paper, we consider one of the preselected scenarios in [10]: an uplink Ka-band FSS cognitive system reusing frequency bands of Fixed-Service (FS) terrestrial microwave links (incumbent systems), as depicted in Fig. 1. In this scenario, the FSS terminal users can maximize frequency exploitation by flexible utilization of the FS segment through the adoption of CR techniques in the satellite uplink. Therefore, this scenario falls within the underlay CR paradigm [14], whereby the uplink power density of the FSS system has to ensure that the interference impact on the potentially present FS links does not exceed the regulatory interference limitations. The applicability of $\mathrm{CR}$ in the aforementioned scenario was discussed in $[15,16]$, considering realistic channel propagation conditions, concluding that both satellite and terrestrial systems could potentially operate in the same band without degrading each other's performance. Here, we go a step further, and consider designing efficient resource allocation algorithms for this scenario. Our goal is to optimally assign carriers and adjust the transmit power of the cognitive devices so that the individual QoS requirement is satisfied and the interference 
at each of the FS stations is kept below the given threshold. Assuming a FS database-assisted approach in which the interference caused at the incumbent system can be perfectly determined and assuming free space path loss between satellite terminal and terrestrial stations, the proposed approach identifies the worst FS link per user in terms of received interference and divides the amount of tolerable interference among the maximum number of FSS terminal users that might potentially interfere with it. In so doing, a transmit power limit is obtained per user and carrier level. This simple and conservative strategy guarantees the cognitive system to never exceed the prescribed interference threshold. The severe restriction in transmit powers is compensated by an efficient Joint Power and Carrier Allocation (JPCA) module that allocates the available resources so as to maximize the overall satellite system throughput.

The remainder of this paper is structured as follows. Section 2 reviews the regulatory context as applied to the frequency bands under consideration. Section 3 introduces the signal model. The proposed cognitive exploitation framework is described in detail in Section 4. Section 5 provides supporting results based on numerical data. Finally, conclusions are given in Section 6 .

\section{Regulatory Context}

Satellites featuring Ka-band transponders have existed for more than a decade. At ITU-R level, the frequency allocations to the FSS uplink span across the whole $27.5-30 \mathrm{GHz}$, with only $500 \mathrm{MHz}$ available exclusively for FSS terminals and the remainder is shared with terrestrial FS links. In Europe, the CEPT framework provides for FSS Earth stations exemption of individual licensing in certain parts of the Ka-band [17]. CEPT sets the band 29.5-30 GHz for exclusive FSS use (same as ITU) and the rest is segmented into specific portions designated for use by uncoordinated FSS Earth stations and for the use by terrestrial FS links. It should be noted, however, that CEPT decisions are not mandatory instruments, and CEPT administrations may choose not to implement them. Examples of european counties not following this decision are UK, Denmark, Austria, Sweden and Lithuania. At the time being, the FSS satellite system is only allowed to operate in the exclusive $29.5-30 \mathrm{GHz}$ band, which is insufficient to meet future demands. Different research projects $[10,12]$ are investigating whether CR techniques could help resolve this specific sharing scenario. In this respect, this paper investigates FSS cognitive satellite terminals operating in the Ka band 27.5-30 GHz, reusing frequency bands of FS links with priority protection.

\section{Signal Model}

In the Ka band scenario being addressed in this paper, the cognitive FSS terminals might impose interference to the terrestrial FS incumbent system. Let us assume a scenario with $L$ FSS terminal users and $N$ FS microwave links. 
The aggregated interference power caused by the $L$ cognitive transmitters at the $n$-th FS microwave station for a particular carrier frequency $f_{m}, m=1, \ldots, M$, is given by,

$$
I_{n}(m)=\sum_{l=1}^{L} p_{l} \cdot g_{l, n}(m),
$$

where $p_{l}$ denotes the transmit power of the $l$-th FSS terminal and $g_{l, n}(m)=$ $\left|h_{l, n}(m)\right|^{2}$, with $h_{l, n}(m)$ being the average cross-channel coefficient from the $l$-th FSS terminal to the $n$-th FS station when the FSS user is transmitting at $f_{m}$.

This average cross-channel gains $g_{l, n}(m)$ can be seen as the Cross-Channel State Information (CCSI). Throughout this paper, we assume a FS databaseassisted approach in which perfect CCSI is assumed available at the FSS system. Clearly, the accuracy and completeness of the available database determines the quality of the CCSI. In this respect, verification of available database via measurements will be considered in future works. The cross-channel gains $g_{l, n}(m)$ can be expressed as follows,

$$
g_{l, n}(m)=G_{\mathrm{Tx}}^{F S S}\left(\theta_{l, n}\right) \cdot G_{\mathrm{Rx}}^{\mathrm{FS}}\left(n, \theta_{n, l}\right) \cdot L\left(d_{l, n}, f_{m}\right)
$$

where,

- $G_{\mathrm{Tx}}^{F S S}(\theta)$ : Gain of the FSS transmitting antenna at offset angle $\theta$.

$-\theta_{i, j}$ : Offset angle (from the boresight direction) of the $i$-th station in the direction of the $j$-th station.

- $G_{\mathrm{RX}}^{\mathrm{FS}}(n, \theta)$ : Gain of the $n$-th FS station antenna at offset angle $\theta$.

- $L(d, f)=\left(\frac{c}{4 \pi d f}\right)^{2}$ : Free space path loss with $d$ being the transmitter-receiver distance and $f$ being the carrier frequency.

- $d_{i, j}$ : Distance between the $i$-th transmitter and the $j$-th receiver.

The radiation patterns $G_{\mathrm{TX}}^{\mathrm{FSS}}(\theta)$ and $G_{\mathrm{Rx}}^{\mathrm{FS}}(n, \theta)$ can be obtained from ITU-R S.465-6 and ITU-R F.1245-2, respectively. Unlike [15,16], we consider the worst case propagation model that would result from a line-of-sight path through free space, with no obstacles nearby to cause reflection or diffraction. In (2), it is assumed that the interfering signal falls within the victim bandwidth. If the spectra do not overlap completely, then a compensation factor of $B_{\text {overlap }} / B^{\mathrm{FS}}$ is applied, where $B_{\text {overlap stands for the portion of the interfering signal spectral }}$ density within the receive filter bandwidth given by $B^{\mathrm{FS}}$.

Satisfying Quality of Service (QoS) requirements of the incumbent FS link requires guaranteeing received interference less than a tolerable level $I_{\mathrm{thr}, n}$. This protection condition is expressed mathematically as,

$$
I_{n}(m) \leq I_{\mathrm{thr}, n}
$$

Such limitations are defined by the regulatory authorities. Typical reference limitations are given by ITU recommendations such as ITU-R F.758, where the interference level is recommended to be $-10 \mathrm{~dB}$ below the receiver noise. 


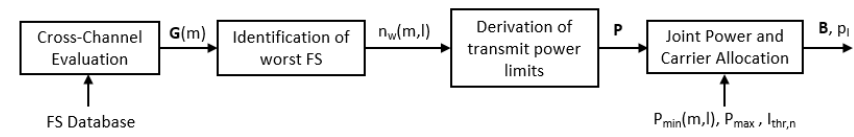

Fig. 2: Block diagram of the cognitive exploitation framework.

Apart from the transmit power limits given by the interference constraints, each FSS terminal has its own power limits. According to CEPT, the maximum EIRP of FSS terminals shall not exceed a value in a range from $55 \mathrm{dBW}$ to 60 dBW. As far as cognitive QoS is concerned, a minimum signal-to-interference and noise ratio (SINR) shall be guaranteed for correct decoding. Therefore, $P_{\min }(m, l) \leq p_{l} \leq P_{\max }$ where $P_{\max }$ denotes the maximum allowable transmission power due to regulatory issues and $P_{\min }(m, l)$ denotes the minimum required power for $l$-th FSS user to close the link over the $m$-th carrier.

\section{Cognitive Exploitation Framework}

One of the major challenges for cognitive uplink satellite communications is, thus, how to optimally assign carriers and adjust the transmitted power so that the individual QoS requirement is satisfied and the aggregate interference at each of the FS stations is kept below the given limit. The level of received interference at each FS station depends both on the transmitted powers $p_{l}, l=1, \ldots, L$, and also on the carrier assignment. Therefore, power and carrier allocation should be considered jointly, which lead to cumbersome optimization problems [18].

In this paper, we follow a simple, but efficient approach in which the transmit power and the assigned carrier of the FSS terminals are determined based on the worst cross-channel condition. The proposed approach first evaluates the cross-channel gains at the carrier level based on the available information at the FS database. With this information, the Network Controller (NC) of the FSS systems is able to identify the worst FS link in terms of received interference per user and per carrier. Next, the amount of tolerable interference of the worst incumbent FS link is divided among the maximum number of FSS terminals that can potentially contribute to its aggregate interference. Subsequently, the maximum transmitted power of each FSS station is derived per each carrier and user. The resulting powers are fed to the JPCA module in order to allocate the resources by maximizing the overall throughput of the FSS system. The schematic diagram of the cognitive exploitation framework is depicted in Fig. 2. Next sections are devoted to describe in detail the proposed cognitive exploitation blocks.

\subsection{Cross-Channel Evaluation and Identification of Worst FS Receiver}

Having complete information on FS database allows the $\mathrm{NC}$ to compute the cross-channel gains $g_{l, n}(m), l=1, \ldots, L, n=1, \ldots, N, m=1, \ldots, M$, as in (2). 
Therefore, for each carrier frequency, the cross-channel matrix $\mathbf{G}(m) \in \mathbb{R}^{L \times N}$ can be described as follows,

$$
\mathbf{G}(m)=\left[\begin{array}{ccc}
g_{1,1}(m) & \cdots & g_{1, N}(m) \\
\vdots & \ddots & \vdots \\
g_{L, 1}(m) & \cdots & g_{L, N}(m)
\end{array}\right] .
$$

For each $l$-th FSS user, the identification of the worst FS station in terms of interference consists in determining the one with maximum cross-channel gain, $n_{\mathrm{w}}(m, l)=\max _{n}[\mathbf{G}(m)]_{l}$, where $[\mathbf{G}(m)]_{l}$ denotes the $l$-th row of matrix $\mathbf{G}(m)$ and $n_{\mathrm{w}}(m, l)$ indicates the worst FS station of user $l$ operating in carrier $m$. These worst FS stations in terms of received interference will determine the maximum allowable transmit power per user.

\subsection{Transmit Power Limits}

The first step is to obtain the interference power limit for each FSS terminal user at a carrier level. For a particular $l$ FSS user, the interference limit of the worst FS receiver, $I_{\mathrm{thr}, n_{\mathrm{w}}(m, l)}[\mathrm{W}]$, is broken into different portions according to the maximum number of FSS users that can potentially interfere with it. This is,

$$
I_{\mathrm{w}}(m, l)=I_{\mathrm{thr}, n_{\mathrm{w}}(m, l)}\left(\frac{B^{\mathrm{FS}}}{B^{\mathrm{FSS}}}\right)^{-1} .
$$

where the fraction $\frac{B^{\mathrm{FS}}}{B^{\mathrm{FSS}}}$ gives the maximum number of FSS terminals that fit in the FS frequency band. Therefore, the transmit power limit is established to ensure that the following individual interference constraint is satisfied,

$$
I_{\mathrm{w}}(m, l) \leq p_{l} \cdot G_{\mathrm{Tx}}^{F S S}\left(\theta_{l, n}\right) \cdot G_{\mathrm{Rx}}^{\mathrm{FS}}\left(n, \theta_{n, l}\right) \cdot L\left(d_{l, n}, f_{m}\right) .
$$

As a consequence, we can obtain the maximum transmission power that FSS terminals should not exceed to guarantee the incumbent system protection in the following way,

$$
p_{\max }(m, l)=\frac{I_{\mathrm{w}}(m, l)}{G_{\mathrm{Tx}}^{F S S}\left(\theta_{l, n}\right) \cdot G_{\mathrm{Rx}}^{\mathrm{FS}}\left(n, \theta_{n, l}\right) \cdot L\left(d_{l, n}, f_{m}\right)} .
$$

Note that there could be some frequencies where no FS is deployed leading to $p_{\max }(m, l) \rightarrow \infty$ or very good conditions in which $p_{\max }(m, l)>P_{\max }$. Moreover, we might face the opposite situation in which the interference constraint is too strong and the value of $p_{\max }(m, l)$ is below the minimum required power. To overcome this infeasibility conditions, the resulting $p_{\max }(m, l)$ are subject to the following adjustments,

$$
p(m, l)= \begin{cases}P_{\max } & \text { if } p_{\max }(m, l)>P_{\max } \\ p_{\max }(m, l) & \text { if } P_{\min }(m, l) \leq p_{\max }(m, l)<P_{\max } \\ 0 & \text { otherwise }\end{cases}
$$


For notational convenience, we define $\mathbf{P} \in \mathbb{R}^{M \times L}$ as the matrix containing the maximum allowable powers due to interference constraint,

$$
\mathbf{P}=\left[\begin{array}{ccc}
p(1,1) & \cdots & p(1, L) \\
\vdots & \ddots & \vdots \\
p(M, 1) & \cdots & p(M, L)
\end{array}\right]
$$

Assuming the worst-case interference in which the complete FS receiver bandwidth is shared with cognitive transmitters is a very conservative assumption which, although protects FS terrestrial system to the maximum extent, it might cause undesirable reductions in the FSS system throughput. This loss in performance, however, can be efficiently compensated with proper carrier allocation by favoring uplink transmissions on carriers with better cross-channel conditions.

The conservative power derivation, thus, ensures that any combination of the powers contained in $\mathbf{P}$ never results in an aggregate interference above the acceptable threshold $I_{\mathrm{thr}, n_{\mathrm{w}}(m, l)}$. Next section is devoted to optimally choose a power and carrier combination from $\mathbf{P}$ that maximizes the sum-rate of the FSS system.

\subsection{Joint Power and Carrier Allocation (JPCA)}

Having tackled the problem of incumbent terrestrial system protection, this section is devoted to optimally allocating the carriers and powers among FSS terminal users by maximizing the total FSS system throughput. We denote $\mathbf{b}_{l} \in \mathbb{R}^{M \times 1}$ the carrier assignment of $l$-th FSS user. The elements of $\mathbf{b}_{l}$ work as an indicator function: " 1 " if $m$-th carrier is assigned to the $l$-th user and " 0 " otherwise. For notational convenience, we stack all the carrier assignments in the matrix $\mathbf{B}=\left[\mathbf{b}_{1} \cdots \mathbf{b}_{L}\right]$. Here, we assume that the FSS users are allocated within the same beam, and each carrier includes a frame which can accommodate a maximum number of users. However, for simplifying the description of the resource allocation module, we assume herein that each carrier can be assigned to only one user. Extension to the case where a given number of users share the same carrier using time division multiplexing (MF-TDMA or Mx-DMA) is then straightforward. Since we assume that at each time, only one user can use a carrier, thus for each carrier $m$, we have $\forall m: \sum_{l=1}^{L} b(m, l)=1$. Note that having obtained the carrier allocation matrix $\mathbf{B}$, it is straightforward to compute the corresponding power allocation as $p_{l}=\mathbf{b}_{l}^{H} \mathbf{p}_{l}$, where $\mathbf{p}_{l}$ stands for the $l$-th column of $\mathbf{P}$.

As discussed in the previous section, the information regarding the maximum allowable power for each user over each available carrier is available in the NC. This information is used to determine the value of $b(m, l)$ to be zero or one by maximizing the overall throughput of the system according to the following optimization problem,

$$
\max _{\mathbf{B}}\|\operatorname{vec}(\mathbf{B} \odot \mathbf{R}(\mathbf{S I N R}))\|_{l_{1}} \quad \text { s.t. } \quad \sum_{l=1}^{L} b(m, l)=1,
$$


where $\odot$ denotes the Hadamard product, $\operatorname{vec}(\cdot)$ denotes the vectorization operator, $\|\cdot\|_{l_{1}}$ denotes the $l_{1}$-norm and $\mathbf{R}(\mathbf{S I N R})$ denotes the rate matrix with $r_{l, m}, l=1, \ldots, L, m=1, \ldots, M$, elements indicating the DVB-S2X rate [19] associated with the corresponding SINR value. In (10), SINR $\in \mathbb{R}^{M \times L}$ denotes the SNR values derived from $\mathbf{P}$ as follows,

$$
\operatorname{SNR}(m, l)=\frac{p(m, l) \cdot G_{\mathrm{Tx}}^{\mathrm{FSS}}(0) \cdot[G / T]_{\mathrm{Rx}}^{\mathrm{SAT}}(l) \cdot L\left(D, f_{m}\right)}{\mathrm{k} B^{\mathrm{FSS}}},
$$

where $[G / T]_{\mathrm{Rx}}^{\mathrm{SAT}}(l)$ is the satellite gain over noise temperature for the $l$-th FSS terminal user, $G_{\mathrm{Tx}}^{\mathrm{FSS}}(0)$ denotes the FSS terminal antenna gain in the boresight direction $\left(\theta=0^{\circ}\right)$ and $D$ is the distance between the FSS terminal and the satellite. On the denominator, $\mathrm{k}$ is the Boltzmann constant. The SINR values are obtained considering a $[C / I]_{\mathrm{Rx}}^{\mathrm{SAT}}$ term which accounts for the co-channel interference.

We solve the optimization problem in (10) using the Hungarian algorithm [20], which provides an efficient and low complexity method to solve the one-toone assignment problem in polynomial time.

\section{Numerical Evaluation}

In this section, we present some numerical results to demonstrate the performance of the proposed JPCA technique to give Ka-band cognitive uplink access to the FSS terminals reusing frequency bands of FS terrestrial microwave links with priority protection.

\subsection{Simulation Setup}

The FS database is extracted from the ITU-R BR International Frequency Information Circular (BR IFIC) database [21]. In particular, we focus on the database related to Slovenia with more than 3,300 records, which is one of the most complete database available for this scenario in BR IFIC. We consider an FSS multibeam satellite system and focus on a representative beam of $155 \mathrm{~km}$ radius with its center located in the capital and largest city of Slovenia, Ljubljana $\left(46.0553^{\circ} \mathrm{N}\right.$ and $\left.14.5144^{\circ} \mathrm{E}\right)$. The beam gain $[G / T]_{\mathrm{Rx}}^{\mathrm{SAT}}(l)$ is computed as $[22]$,

$$
[G / T]_{\mathrm{Rx}}^{\mathrm{SAT}}(l)=[G / T]_{\mathrm{Rx}, \max }^{\mathrm{SAT}} \cdot\left(\frac{J_{1}\left(u\left(\varphi_{l}\right)\right)}{2 u\left(\varphi_{l}\right)}+36 \frac{J_{3}\left(u\left(\varphi_{l}\right)\right)}{u\left(\varphi_{l}\right)^{3}}\right)^{2}
$$

where $[G / T]_{\mathrm{Rx}, \max }^{\mathrm{SAT}}$ is the maximum satellite antenna gain, $J_{p}$ is the first kind of Bessel's function of order $p$ and $u\left(\varphi_{l}\right)=2.07123 \cdot \sin \left(\varphi_{l}\right) / \sin \left(\varphi_{3 \mathrm{~dB}}\right)$, with $\varphi_{l}$ and $\varphi_{3 \mathrm{~dB}}=0.2^{\circ}$ being the satellite nadir angle corresponding to user $l$ and the half-power beamwidth, respectively.

The performance was evaluated by averaging over 50 independent FSS terminal geographical distributions, which were selected uniformly at random for 
each realization within the considered beam footprint according to the population density database obtained from the NASA Socioeconomic Data and Applications Center (SEDAC) [23]. The number of FSS terminals $L$ is set to be 356, which coincides with the number of carriers to be assigned. A summary of the most relevant parameters and the FSS link budget details are presented in Table 1. An example of FSS terminal users distribution together with the location of the FS stations and the beam pattern of the FSS satellite obtained with (12) is depicted in Fig. 3.

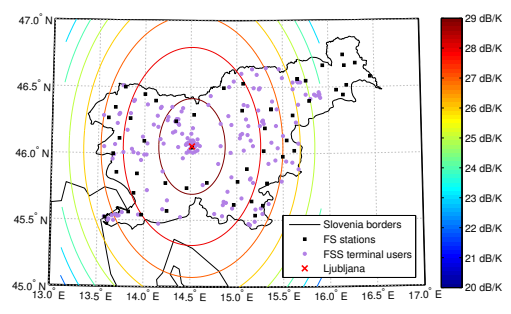

Fig. 3: Beam pattern of the FSS satellite over Slovenia.

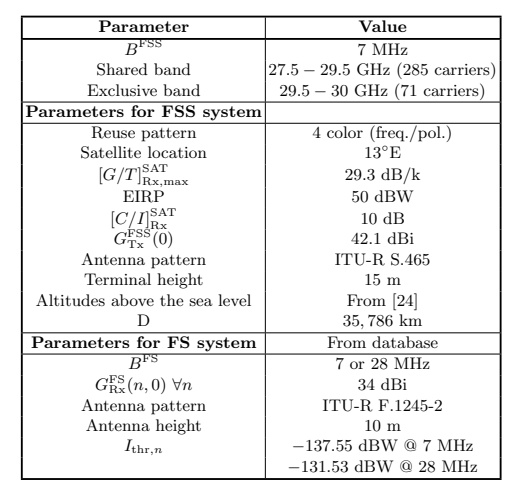

Table 1: Simulation Parameters

\subsection{Numerical Results}

The spectrum occupancy of the BRIFIC FS database is depicted in Fig. 4, which shows that around $60 \%$ of the shared spectrum band (27.5 to $29.5 \mathrm{GHz}$ ) is occupied in Slovenia. Thus, without any additional work, the uplink band could benefit from more than $800 \mathrm{MHz}$ of additional bandwidth.

Fig. 5 shows the aggregate interference caused by the cognitive FSS system and received at the FS stations in terms of Cumulative Distribution Function $(\mathrm{CDF})$ with and without the proposed JPCA module. For the latter, a random carrier assignment is considered and $p_{l}=P_{\max }, \forall l$. For the JPCA case, the optimal and one sub-optimal combination of the powers contained in $\mathbf{P}$ are plotted in Fig. 5. The minimum aggregate interference threshold is depicted as well in the figure for comparison purposes. It can be observed that the interference generated by the FSS system at the incumbent system exceeds the acceptable threshold when no optimal resource assignment is employed and it is kept always below the threshold when using the proposed JPCA technique.

The effect of the transmit power limit given by the interference constraint (3) is evident by a glance of Fig. 6, where the SINR of the FSS terminal users is shown in terms of CDF with optimal, suboptimal and without JPCA. An immediate observation is that the SINR degrades when transmit power limitations 


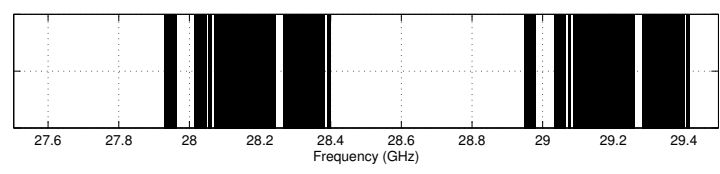

Fig. 4: Spectrum occupancy map of Slovenia. Black means occupied by FS system.

apply, i.e., when FS terrestrial microwave links are protected. In particular, 35\% of FSS users experience SINR values below $9.8 \mathrm{~dB}$ in the FSS-FS coexistence case when resources are not allocated optimally, which decreases down to $22.5 \%$ if the proposed JPCA is employed, and to $9.3 \%$ when the transmit power is not limited (blue line).

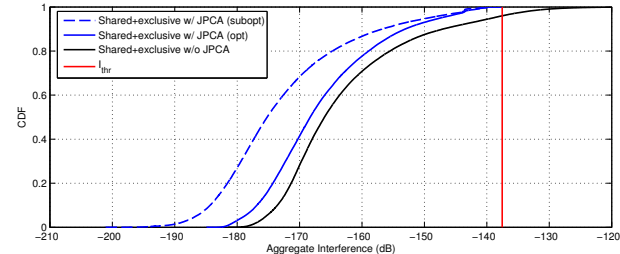

Fig. 5: CDF of aggregate interference

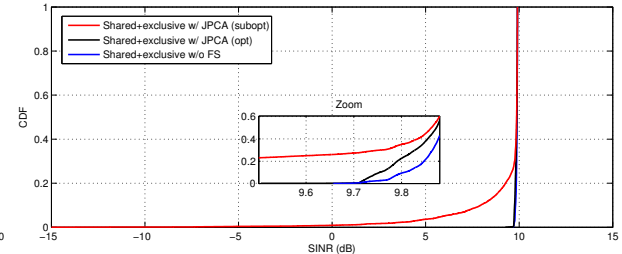

Fig. 6: CDF of SINR distribution

Similarly, Fig. 7 presents the comparison of rate distribution in the considered scenario. From the figure, it can be noted that the beam availability in the presence of the incumbent FS system is less than the beam availability when no power constraints apply (blue line). Further, it is clear that employing the proposed JPCA attains a throughput per user that is very close to that attained in the absence of transmit power limitations.

Fig. 8 illustrates the achieved per beam throughput. To obtain greater insight, Table 2 summarizes the results shown in Fig. 8. It is worth to point out that the additional spectrum together with the optimal JPCA module provides around $405.8 \%$ improvement over the conventional exclusive band (29.5-30 GHz) case, which is almost the same that can be achieved in the absence of FS microwave links. This gain reduces to $378.6 \%$ when resources are not optimally distributed. The application of optimal JPCA in the non-shared spectrum scenarios does not provide much benefit and this is because, in this particular study, all users and carriers experience similar channel conditions.

\section{Conclusion}

In this paper, we developed a novel spectrum exploitation framework for cognitive uplink FSS terminals in the band $27.5-29.5 \mathrm{GHz}$, where the incumbent 


\begin{tabular}{|c|c|c|}
\hline Case & Technique & Value (Mbps) \\
\hline \multirow{2}{*}{ Exclusive only } & w/ JPCA (subopt) & 699.5136 \\
\cline { 2 - 3 } & w/ JPCA (opt) & 699.5291 \\
\hline \multirow{2}{*}{ Shared+Excl. w/o FS } & w/ JPCA (subopt) & 3538.0503 \\
\cline { 2 - 3 } & w/ JPCA (opt) & 3538.5299 \\
\hline \multirow{2}{*}{ Shared+Excl. w/ FS } & w/ JPCA (subopt) & 3347.6373 \\
\cline { 2 - 3 } & w/ JPCA (opt) & 3538.1431 \\
\hline
\end{tabular}

Table 2: Throughput per beam

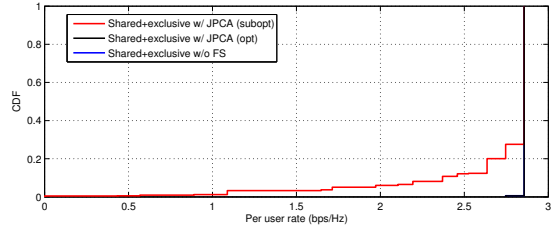

Fig. 7: Per user rate

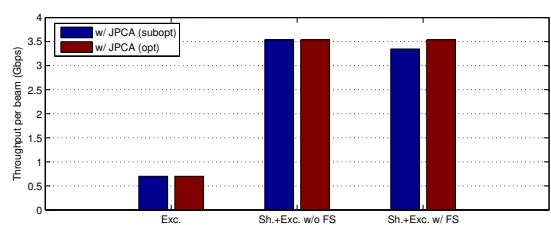

Fig. 8: Throughput per beam

users are Fixed-Service (FS) microwave links. Results based on computer simulations were presented, which showed that the FSS system throughput can be significantly improved by using the proposed cognitive exploitation framework while guaranteeing the sufficient protection of incumbent FS systems.

\section{Acknowledgment}

This work was partially supported by the European Commission in the framework of the FP7 Cognitive Radio for SATellite communications - CoRaSat (Grant agreement no. 316779) and by National Research Fund, Luxembourg, under CORE project SpEctrum Management and Interference mitiGation in cognitive raDio satellite networks - SeMIGod, and CORE project SATellite SEnsor NeTworks - SATSENT.

\section{References}

1. EU, "Digital agenda for europe," http://ec.europa.eu/digital-agenda, [Online; accessed 8-Dec-2014].

2. European Commission, "A Digital Agenda for Europe," COM(2010)245, Brussels, Belgium, May, 2010.

3. D. Brandel, W. Watson, and A. Weinberg, "NASA's Advanced Tracking and Data Relay Satellite System for the Years 2000 and Beyond," Proceedings of the IEEE, vol. 78, no. 7, pp. 1141-1151, Jul, 1990.

4. R. Alegre-Godoy, N. Alagha, and M. Vazquez-Castro, "Offered Capacity Optimization Mechanisms for Multi-Beam Satellite Systems," IEEE Int. Conf. on Communications (ICC), Ottawa, Canada, Jun, 2012.

5. EUTELSAT, http://www.eutelsat.com, [Online; accessed 9-Dec-2014]. 
6. VIASAT, https://www.viasat.com, [Online; accessed 9-Dec-2014].

7. SES, https://www.ses.com, [Online; accessed 7-Jan-2015].

8. M. Aloisio, P. Angeletti, F. Coromina, D. Mignolo, D. Petrolati, and E. Re, "A System Study to Investigate the Feasibility of Terabit/s Satellites," IEEE Int. Vacuum Electronics Conf. (IVEC), Monterey, CA, USA, Apr, 2012.

9. S. Haykin, "Cognitive radio: brain-empowered wireless communications," IEEE Journal on Selected Areas in Communications, vol. 23, no. 2, pp. 201-220, 2005.

10. "COgnitive RAdio for SATellite Communications - CoRaSat," European Commission FP7, Oct, 2012.

11. "Cooperative and Cognitive Architectures for Satellite Networks - CO2Sat," Fonds National de la Recherche Luxembourg (FNR), 2011.

12. "Spectrum Management and Interference Mitigation in Cognitive Radio Satellite Networks - SeMiGod," Fonds National de la Recherche Luxembourg (FNR), 2014.

13. "Antennas and Signal Processing Techniques for Interference Mitigation in Next Generation Ka Band High Throughput Satellites - ASPIM," European Space Agency, Sep, 2014.

14. A. Goldsmith, S. A. Jafar, I. Maric, and S. Srinivasa, "Breaking Spectrum Gridlock with Cognitive Radio: An Information Theoretic Perspective," Proc. IEEE, vol. 97, no. 5, pp. 894-914, May, 2009.

15. A. Mohamed, M. Lopez-Benitez, and B. Evans, "Ka Band Satellite Terrestrial Co-Existence: A Statistical Modelling Approach," Ka and Broadband Communications, Navigation and Earth Observation Conf., Salerno, Italy, Oct, 2014.

16. S. Maleki, S. Chatzinotas, B. Evans, K. Liolis, J. Grotz, A. Vanelli-Coralli, and N. Chuberre, "Cognitive Spectrum Utilization in Ka Band Multibeam Satellite Communications," to appear in IEEE Communication Magazine.

17. "The use of the band $27.5-29.5 \mathrm{GHz}$ by the Fixed Service and uncoordinated Earth stations of the Fixed-Satellite Service (Earth-to-space)," ECC Decision $E C C / D E C /(05) 01$, Mar, 2005.

18. M. Hong and Z. Luo, "Signal Processing and Optimal Resource Allocation for the Interference Channels," in Academic Press Library in Signal Processing: Communications and Radar Signal Processing, Chellappa, R. and Theorodiris, S., Ed. Oxford, UK: Elsevier, 2014.

19. Digital Video Broadcasting (DVB), "DVB-S2X Standard," https://www.dvb.org/ standards/dvb-s2x, [Online; accessed 7-Oct-2014].

20. H. Kuhn, "The Hungarian Method for the Assignment Problem," Naval Research Logistics Quarterly, vol. 2, pp. 83-97, 1955.

21. ITU, "Br ific for terrestrial services," http://www.itu.int/en/ITU-R/terrestrial/ brific, [Online; accessed 7-Oct-2014].

22. C. Caini, G. Corazza, G. Falciasecca, M. Ruggieri, and F. Vatalaro, "A Spectrum and Power Efficient EHF Mobile Satellite System to be Integrated with Terrestrial Cellular Systems," IEEE J. Sel. Areas Commun., vol. 10, no. 8, pp. 1315-1325, 1992.

23. NASA, "Socioeconomic Data and Applications Center (SEDAC)," http://sedac. ciesin.columbia.edu, [Online; accessed 27-Feb-2015].

24. USA, "US National Geospatial-Intelligence Agency (NGA)," http://geoengine.nga. mil/muse-cgi-bin/rast_roam.cgi, [Online; accessed 18-Feb-2015]. 